OPEN ACCESS

Edited by:

Renato Franco,

University of Campania Luigi

Vanvitelli, Italy

Reviewed by:

Tatjana Vlajnic,

Institute of Pathology, University

Hospital Basel, Switzerland

Thomas Menter,

University Hospital of Basel,

Switzerland

*Correspondence:

Chunguang Yang

cgyang-hust@hotmail.com

†These authors have contributed equally to this work

Specialty section:

This article was submitted to

Pathology,

a section of the journal

Frontiers in Medicine

Received: 11 May 2020

Accepted: 06 July 2020

Published: 27 August 2020

Citation:

Hu Z, Ke C, Liu Z, Zeng X, Li S, Xu H and Yang $C$ (2020) Evaluation of UroVysion for Urachal Carcinoma Detection. Front. Med. 7:437. doi: 10.3389/fmed.2020.00437

\section{Evaluation of UroVysion for Urachal Carcinoma Detection}

\author{
Zhiquan $\mathrm{Hu}^{\dagger}$, Chunjin $\mathrm{Ke}^{\dagger}$, Zheng Liư ${ }^{\dagger}$, Xing Zeng, Song Li, Hua Xu and Chunguang Yang* \\ Department of Urology, Tongji Hospital Affiliated to Tongji Medical College of Huazhong University of Science and Technology \\ (HUST), Wuhan, China
}

Background: Patients with hematuria who are positive for urinary fluorescence in situ hybridization (FISH) are generally considered to have urothelial carcinoma. We determined whether UroVysion FISH could be used for the diagnosis of urachal carcinoma.

Methods: Seven cases of urachal carcinoma with haematuria subjected to FISH analysis were retrospectively analyzed in our hospital from May 2012 to November 2019. Paraffin-embedded tissue sections from one FISH-positive and one FISH-negative urachal carcinoma were processed in strict accordance with the instructions of the UroVysion kit. Meanwhile, FISH data from the other 414 hematuria patients were collected as controls.

Results: All 7 patients with urachal carcinoma were diagnosed with adenocarcinoma. According to Sheldon stage, six patients had stage IIla and one patient had stage IVb. The sensitivity and specificity of urinary FISH for the diagnosis of urachal carcinoma were $71.43 \%(5 / 7)$ and $94.61 \%$ (281/297), respectively. The rates of polysomy for chromosomes 3 and 7 in positive patients were both 100\% (5/5), whereas the rate of polysomy for chromosome 17 was 40\% (2/5), and the chromosome 9p21 region (p16) gene deletion rate was 20\% (1/5). Histological assessment and cytological FISH were consistent for urachal carcinoma. No significant difference was observed in the diagnostic efficacy between urachal carcinoma and urothelial carcinoma (71.43 vs. $87.18 \%, P=0.245)$.

Conclusions: Taken together, UroVysion FISH was found to be positive in a high proportion of pathologically confirmed urachal carcinoma of late stage with hematuria. Its chromosomal aberrations may be different from those of urothelial carcinoma, but more studies are needed to clarify their genetic background. Not all tumors showing abnormalities by FISH are urothelial carcinomas.

Keywords: urachal carcinoma, urothelial carcinoma, fluorescence in situ hybridization, UroVysion, differential diagnosis

\section{INTRODUCTION}

The urachus is a tubular structure extending from the top of the bladder to the umbilicus during embryonic development. Before birth and in infancy, the tubular structure disappears and degenerates into the median umbilical ligament, which is located in the median umbilical fold (1). Urachal carcinoma is a rare genitourinary tumor originating from the urachal tract, with an annual 
incidence of $\sim 1 / 5,000,000$ (2). This cancer accounts for 0.35 to $0.7 \%$ of all bladder cancers $(3,4)$. Although the incidence of the disease is low, it is difficult to effectively control the tumor by surgical resection or systemic treatment, and patient safety is seriously threatened. Therefore, preoperative diagnosis is particularly important.

UroVysion fluorescence in situ hybridization (FISH) is a sensitive and specific method for the diagnosis of urothelial carcinoma. The fluorescently labeled DNA probe is denatured into a single strand, after which it hybridizes with the denatured chromosome or nuclear target DNA. The DNA is then observed under a fluorescence microscope. This method has been approved for the screening of patients with hematuria and the monitoring of recurrent urothelial carcinoma. Moreover, many studies have been performed using the application of FISH in urothelial carcinoma (5-10). Thus, patients with hematuria who are positive for urinary FISH are often diagnosed with urothelial carcinoma.

Kipp et al. (11) retrospectively assessed non-urothelial carcinoma (including urachal carcinoma) for chromosomal abnormalities in paraffin tissue sections by FISH with the UroVysion probe set and found that chromosomal abnormalities in urothelial carcinoma are also common in rarer histological variants of bladder cancer. Yang et al. (12) investigated the value of FISH in bladder paraganglioma using urine specimens. In these studies, diagnostic efficacy of UroVysion for rarer histological cancer was not demonstrated. Moreover, the results were not compared between paraffin tissue sections or urine cytology specimens. Our previous clinical work showed that urinary FISH can also show positive signs in urachal carcinoma, which motivated this research. Therefore, this study focused on the diagnostic value of FISH in patients with urachal carcinoma and compared the consistency of histological and cytological FISH results.

\section{PATIENTS AND METHODS}

\section{Patients and Samples}

After the approval of the Department Review Committee, seven cases of urachal carcinoma with urinary FISH analysis data were collected from the medical records department of our hospital from May 2012 to November 2019 (see Table 1 for general clinical information) and were included in this study. The inclusion criteria were as follows: (1) patients with urachal carcinoma confirmed by pathology and clinical data; (2) patients underwent a urinary FISH test before surgery; and (3) systemic treatment was administered after surgery. The exclusion criteria were as follows: patients with a history of malignant tumors or urinary tract infections. At the same time, data on the other 414 patients who underwent FISH testing because of hematuria in our hospital were collected, and these patients served as the control group. Among them, 117 cases were diagnosed with urothelial carcinoma, and 297 cases were diagnosed with non-urothelial tumors. In addition, the paraffin sections of the aforementioned cases A and F were submitted to the pathology department for histological FISH analysis.

\section{FISH Processing}

The FISH DNA probe was provided by Beijing Jinpujia Medical Technology Co., Ltd., and consists of two combinations of Chromosome Region-Specific Probe 3[CSP3 (green)]/CSP7 (red) and gene locus-specific probe p16[GLPp16 (red)]/CSP17 (green). The main reagents needed in the experimental operation are prepared and used in strict accordance with the instructions of FISH, which are mainly provided by Beijing Jinpu Jia Medical Technology Co., Ltd., and the supporting reagents are provided by our hospital reagent room supply (Supplementary Material for details). First, the 5- $\mu \mathrm{m}$ paraffin-embedded sections were placed in a $56^{\circ} \mathrm{C}$ oven overnight and were then placed in xylene for multiple deparaffinization steps. The slides were dehydrated in $100 \%$ ethanol for $5 \mathrm{~min}$ at room temperature, and after air drying for $3 \mathrm{~min}$, the slides were immersed in $10 \mathrm{mmol} / \mathrm{L}$ citric acid buffer $\left(80^{\circ} \mathrm{C}\right.$; $\left.\mathrm{pH} 6.0\right)$ for $45 \mathrm{~min}$, followed by immersion in $2 \times$ saline-sodium citrate (SSC) for $5 \mathrm{~min}$ at $37^{\circ} \mathrm{C}$. The samples were then digested in $0.2 \%$ pepsin solution $(2,500-3,500 \mathrm{U} / \mathrm{mg})$ for $48 \mathrm{~min}$ at $37{ }^{\circ} \mathrm{C}$, after which the sections were placed in 70 , 85 , and $100 \%$ ethanol solutions. Each section was dehydrated in ethanol for $2 \mathrm{~min}$. Next, $10 \mu \mathrm{L}$ of the UroVysion probe (Beijing Jinpujia Medical Technology Co., Ltd) was applied to the target tissue area. The probe and target DNA were denatured and hybridized on a HYBrite instrument at $80^{\circ} \mathrm{C}$ for $3 \mathrm{~min}$ and hybridized at $37^{\circ} \mathrm{C}$ for 16 to $18 \mathrm{~h}$. After hybridization, any unbound probe was removed by washing in $2 \times \mathrm{SSC} / 0.1 \% \mathrm{NP}-$ 40 at $76^{\circ} \mathrm{C}$ for $2 \mathrm{~min}$, followed by $1 \mathrm{~min}$ at room temperature in $2 \times \mathrm{SSC} / 0.1 \% \mathrm{NP}-40$. Then, $10 \mu \mathrm{L}$ of DAPI II counterstain was applied, and the slides were cover-slipped. Fluorescence in situ hybridization results were analyzed by two professional certified double-blind pathologists with 10 years of work experience. A minimum of 25 tumor cells were visualized and evaluated for these chromosomal changes. If no abnormalities were detected, then the remaining cells were counted until a sufficient number of cells with chromosomal abnormalities were found or until 200 cells were evaluated. A positive result was the presence of $\geq 4$ (or $>10 \%$ ) cells with gains of 2 or more of chromosomes 3,7 , and 17. In the case of chromosome nine, a positive result was one in which more than 12 cells showed zero 9p21 signals (13).

\section{Statistical Analysis}

SPSS 23.0 was used for the statistical analysis. The statistical data were analyzed with the independent $t$-test and $\chi^{2}$-test. $P<0.05$ was considered statistically significant.

\section{RESULTS}

\section{Application of Urinary FISH for the Detection of Urachal Carcinoma and Urothelial Cancer}

Overall, data on 31 patients with urachal carcinoma were collected from the medical records department, and of them, seven patients met the inclusion and exclusion criteria. According to the Sheldon stage, six of the remaining seven patients had stage IIIa, and one had stage IVb, and the most 
TABLE 1 | General clinical information of 7 patients with urachal carcinoma.

\begin{tabular}{|c|c|c|c|c|c|c|c|}
\hline Patients & Sex & Age (years) & First symptom & FISH (+/-) & Stages & Treatments & Pathology \\
\hline A & Male & 25 & Painless gross hematuria & + & IIla & $\begin{array}{l}\mathrm{EPC}+\text { chemo + radio } \\
+ \text { targeted }\end{array}$ & Medium-differentiated adenocarcinoma \\
\hline$B$ & Male & 54 & $\begin{array}{l}\text { Umbilical blood, purulent } \\
\text { discharge, hematuria }\end{array}$ & + & $\mathrm{IVb}$ & Chemo & Poorly differentiated adenocarcinoma \\
\hline C & Male & 30 & $\begin{array}{l}\text { Painless intermittent } \\
\text { gross hematuria }\end{array}$ & + & IIla & $\mathrm{EPC}+$ chemo + radio & Mucous adenocarcinoma \\
\hline $\mathrm{D}$ & Male & 46 & Gross hematuria & + & IIla & $R C+R B S$ & $\begin{array}{l}\text { Medium/poor-differentiated } \\
\text { adenocarcinoma }\end{array}$ \\
\hline$E$ & Male & 68 & Painless gross hematuria & + & IIla & EPC & Urachal adenocarcinoma \\
\hline $\mathrm{F}$ & Female & 49 & Painless gross hematuria & - & Illa & $\mathrm{RC}+\mathrm{IBS}+\mathrm{PLND}$ & Medium-differentiated adenocarcinoma \\
\hline G & Female & 50 & Gross hematuria & - & IIla & $\mathrm{PC}+$ chemo & Medium-differentiated adenocarcinoma \\
\hline
\end{tabular}

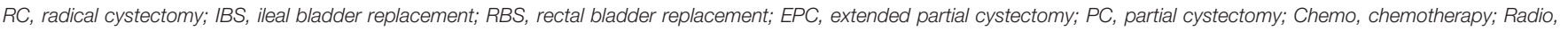
radiotherapy; PLND, pelvic lymph node dissection.

TABLE 2 | Comparison of positive detection rates of urinary FISH in the urachal carcinoma group, urothelial cancer group, and non-urothelial tumor group $[n(\%)]$.

\begin{tabular}{llll}
\hline Groups & FISH & $P$ \\
& & - &
\end{tabular}

\begin{tabular}{lccc}
\hline $\begin{array}{l}\text { Urachal carcinoma group } \\
(n=7)\end{array}$ & $5(71.43)$ & $2(28.56)$ & $P=0.245$ \\
Urothelial carcinoma group & $102(87.18)$ & $15(12.80)$
\end{tabular}

$(n=117)$

Non-urothelial carcinoma $\quad 16(5.40) \quad 281(94.60) \quad{ }^{a} P<0.0001$,

group $(n=297)$

$P=0.245$ refers to the comparison between the urachal carcinoma group and the urothelial carcinoma group. ${ }^{a} P<0.0001$ refers to the comparison between the urachal carcinoma group and the non-urothelial tumor group. The Fisher $\chi^{2}$-test was used. To compare the urothelial carcinoma group with the non-urothelial tumor group, Pearson $\chi^{2}$-test was used, $x^{2}=275.55 .{ }^{b} P<0.0001$.

common symptom was hematuria. The urinary FISH assay was positive in five cases and negative in two cases. The sensitivity and specificity for the diagnosis of urachal cancer were $71.43 \%(5 / 7)$ and $94.61 \%(281 / 297)$, respectively. The control group contained 414 cases, and the urothelial carcinoma group contained 117 cases; 102 and 15 cases of which were positive and negative, respectively. The non-urothelial tumor group included 297 cases, 16 and 281 of which were positive and negative, respectively. It can be seen that the sensitivity and specificity of urinary FISH for the diagnosis of urothelial carcinoma are $87.18 \%$ $(102 / 117)$ and $94.61 \%(281 / 297)$, respectively. No significant difference was observed in the diagnostic efficacy between urachal carcinoma and urothelial carcinoma (71.43 vs. $87.18 \%$, $P=0.245)$ (Table 2).

\section{Chromosome Aberrations in Urinary FISH-Positive Urachal Carcinoma Patients}

The five positive patients were male: four were stage IIIa, and one was stage IVb. The aberration rate of chromosomes 3 and 7 was $100 \%(5 / 5)$, the aberration rate for chromosome 17 was $40 \%$
TABLE 3 | Chromosome aberrations in patients with FISH-positive urachal carcinoma.

\begin{tabular}{|c|c|c|c|c|c|c|}
\hline \multicolumn{3}{|c|}{ Cases sex stages } & \multicolumn{4}{|c|}{ Chromosome polysomy/gene deletion } \\
\hline & & & 3\# & 7\# & $17 \#$ & GLPp16 \\
\hline A & Male & Illa & 1 & 1 & 0 & 0 \\
\hline $\mathrm{B}$ & Male & $\mathrm{IVb}$ & 1 & 1 & 0 & 0 \\
\hline C & Male & Illa & 1 & 1 & 1 & 0 \\
\hline D & Male & Illa & 1 & 1 & 1 & 1 \\
\hline E & Male & Illa & 1 & 1 & 0 & 0 \\
\hline
\end{tabular}

1 , indicates chromosome polysomy or gene deletion; 0 , indicates none.

$(2 / 5)$, and the chromosome $9 \mathrm{p} 21$ region (p16) gene deletion rate was $20 \%(1 / 5)$. Specific abnormal conditions are shown in Table 3 and Figure 1.

\section{Comparison of Histological and Cytological FISH Analysis Results}

Histological FISH performed in case A showed chromosomes 3 and 7 polysomy, no chromosome 17 polysomy, and no chromosome 9p21 region (p16) gene deletion (Figure 2). Histological FISH testing in case F was negative (Figure 3), consistent with cytological FISH results. These findings might indicate that the tumor cells shed into urine originated from tumor tissue.

\section{DISCUSSION}

Although the incidence of urachal carcinoma is very low, it is highly malignant. Patients are usually at a later stage at diagnosis, whereas early urachal carcinoma is often confined to the umbilical duct without obvious clinical symptoms. Clinical symptoms mostly appear when the tumor invades or breaks through the bladder. Hematuria, as the most common symptom of urachal carcinoma (14), is also the most common 


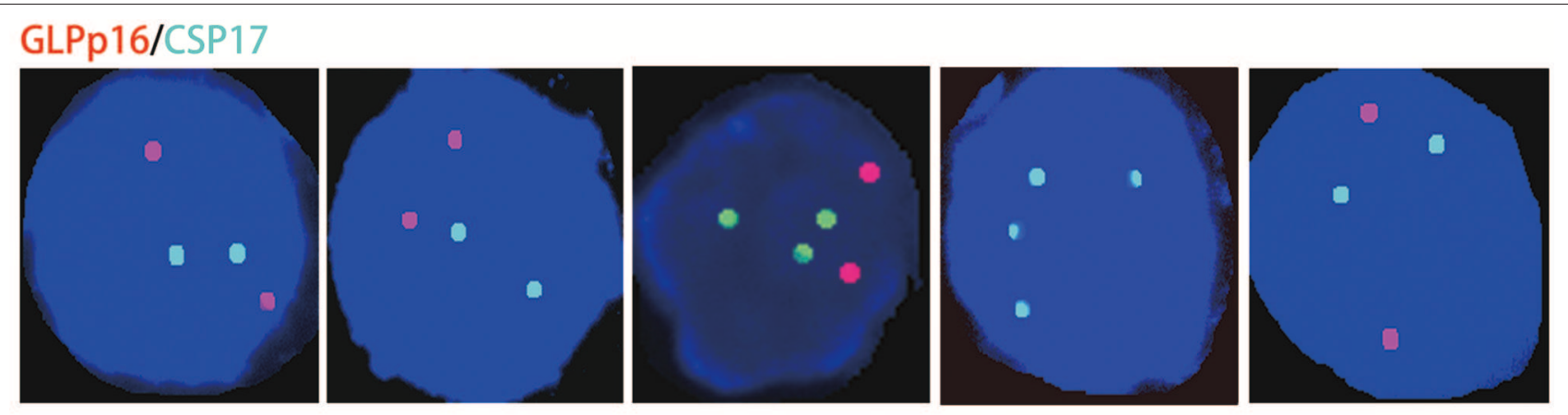

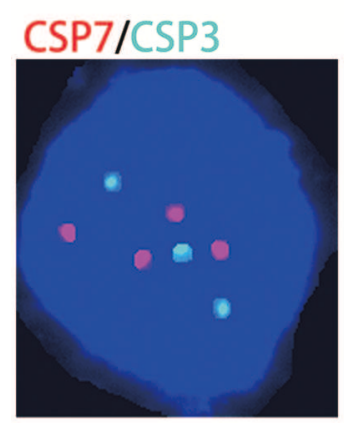

A

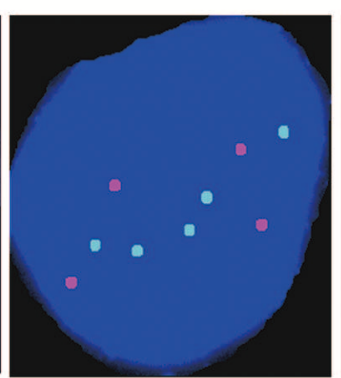

B

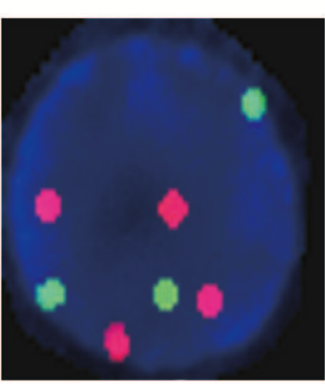

C

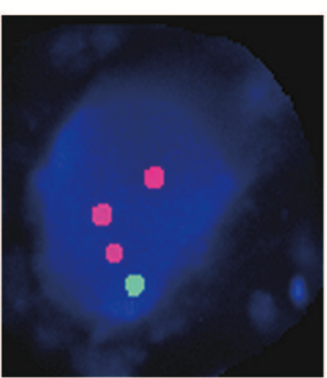

D

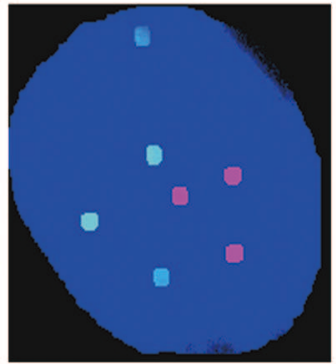

E

FIGURE 1 | Chromosome aberrations in patients with urinary FISH-positive urachal carcinoma. (A-E) Correspond to cases (A-E); red represents CSP7 and GLPp16, whereas green represents CSP7 and CSP17.

symptom of bladder tumors, $\sim 90 \%$ of which are urothelial carcinomas. UroVysion FISH was originally used to diagnose urothelial carcinoma rather than non-urothelial carcinoma. We found that FISH could also yield positive results in urachal carcinoma in previous clinical practice, which motivated this research.

Numerous studies have evaluated the efficacy of FISH in urothelial carcinoma (5-10), whereas data on the evaluation of UroVysion probes in non-urothelial carcinoma are scarce. This study found that the positive rate of FISH in urachal carcinoma of late stage with hematuria was rather high at $71.43 \%$, which was not significantly different from that in urothelial carcinoma ( 71.43 vs. $87.18 \%, P=0.245)$. At the same time, our study showed that the polysomy rate of chromosomes 3 and 7 was $71.43 \%(5 / 7)$, and that of chromosome 17 was $28.57 \%$ $(2 / 7)$. The chromosome 9 p21 region (p16) gene deletion rate was only $14.29 \%(1 / 7)$. However, in a multicenter big data study conducted by Zhou et al. (10) in China, the polysomy of chromosomes 3, 7, and 17 and gene changes at $9 \mathrm{p} 21$ region (p16) accounted for $71.3 \%$ (2941/4125), 72.2\% (2978/4125), $67.4 \%(2780 / 4125)$, and $72.9 \%(3007 / 4125)$ of urothelial carcinoma cases, respectively. Therefore, the chromosome 9p21 region (p16) gene deletion in urachal carcinoma seems to occur less frequently than in urothelial carcinoma (16.67 vs. $72.9 \%, P=0.008)$.
To compare chromosomal aberrations in urine exfoliative cytological FISH and histological FISH, we performed histological FISH of urachal carcinoma, which was not reported previously. Histological FISH in case A also showed chromosomes 3 and 7 polysomy, no chromosome 17 polysomy, and no chromosome 9p21 gene deletion. Histological FISH in case F was negative. Thus, it might indicate that the tumor cells shed in urine originated from urachal carcinoma.

Studies have shown that for patients with invasive and highly malignant tumors, the tumor cells have many genetic abnormalities $(15,16)$. The principle of FISH is to use fluorescently labeled GLPp16 site-specific probes and CSP3/CSP7/CSP17 chromosomal centromere-specific probes that are hybridized in situ with target DNA. If the tumor cells have aberrations in chromosomes $3,7,17$, or 9p21, and the cancerous cells are shed in sufficient quantities into the urine, urinary FISH may be positive in theory. This study also justifies this approach. Kipp et al. (11) and Reid-Nicholson et al. (13) found that metastatic colon cancer, cervical cancer that metastasizes to the bladder, and primary squamous cell carcinoma and small cell carcinoma of the bladder exhibit FISHpositive histological FISH results, which is consistent with our speculation. These results indicate that FISH positivity should not be simply interpreted as urothelial carcinoma. Additionally, 


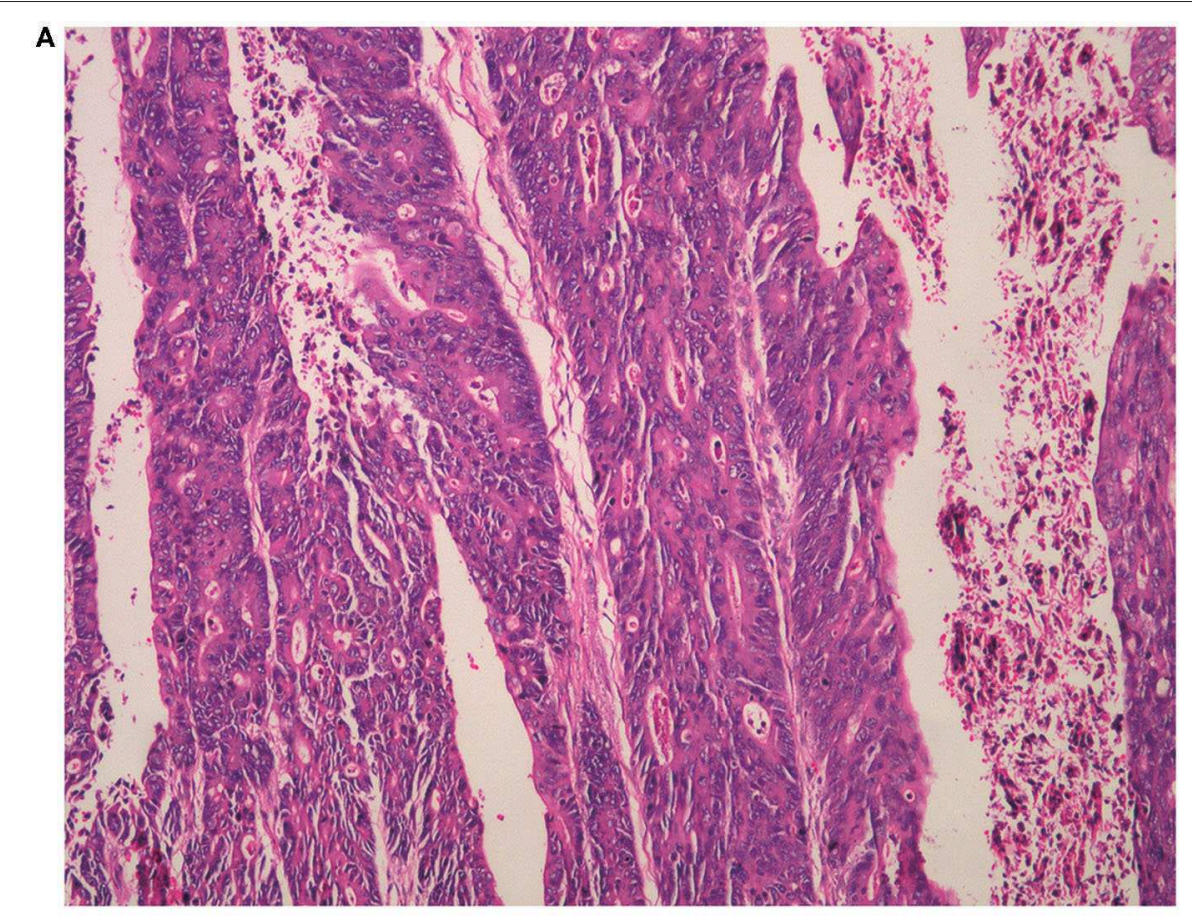

B

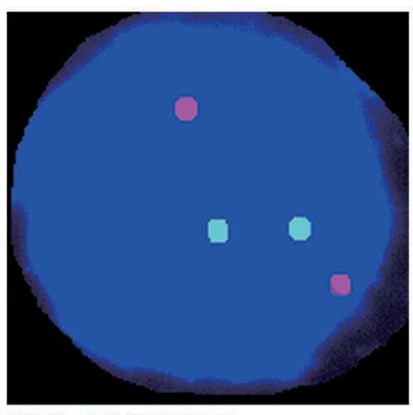

GLPp16/CSP17

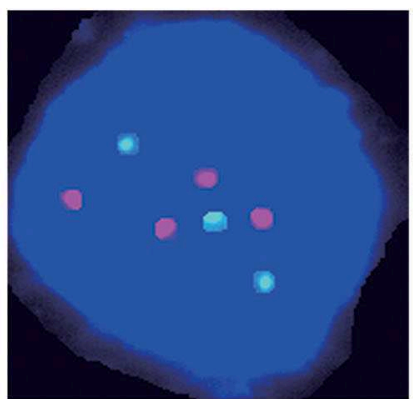

CSP7/CSP3

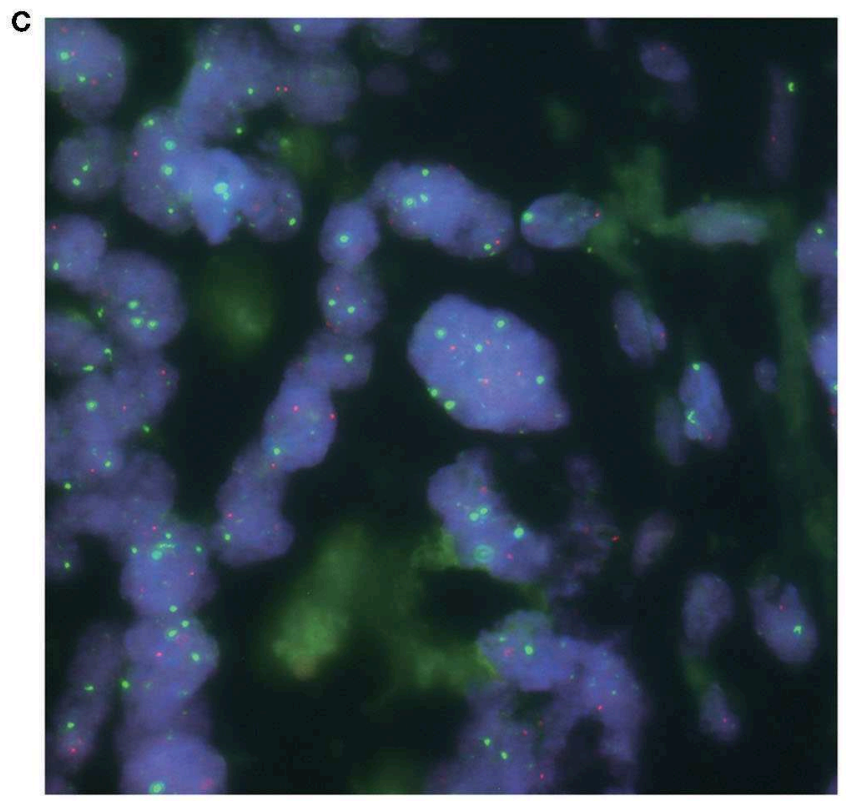

GLPp16/CSP17

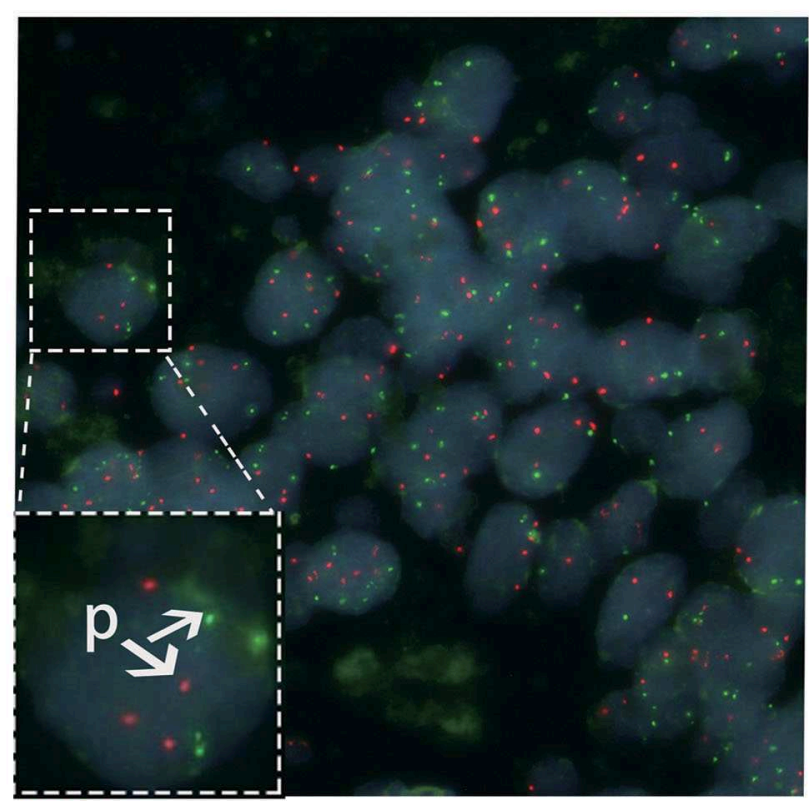

CSP7/CSP3

\section{Case A}

FIGURE 2 | Cytological and histological chromosome aberrations in case A. (A) Microscopy revealed moderately differentiated adenocarcinoma (hematoxylin-eosin staining, magnification $\times 200$ ); (B) case A urinary FISH showed chromosomes 3 and 7 polysomy, no chromosome 17 polysomy, and no chromosome 9p21 region gene deletion; (C) histological FISH in case A also showed chromosomes 3 and 7 polysomy (as shown in P), no chromosome 17 polysomy, and no chromosome 9p21 region gene deletion; red represents CSP7 and GLPp16, and green represents CSP7 and CSP17.

whether the probe can be more scientifically modified to increase its sensitivity and specificity for urachal carcinoma remains to be determined.
Of course, our study has some limitations. Because the incidence of urachal carcinoma is very low, the number of cases is small, and the conclusions should be better verified 

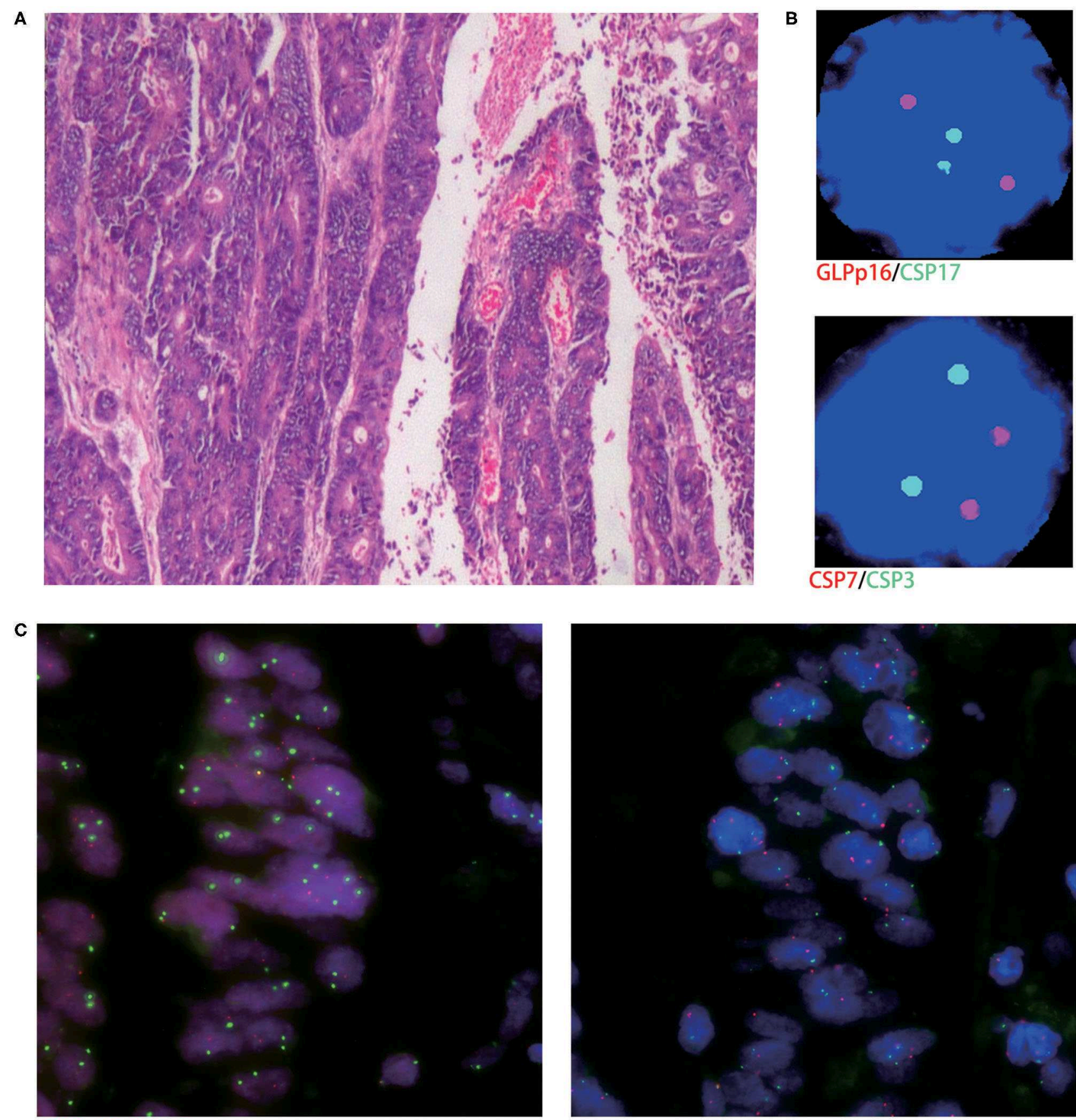

GLPp16/CSP17

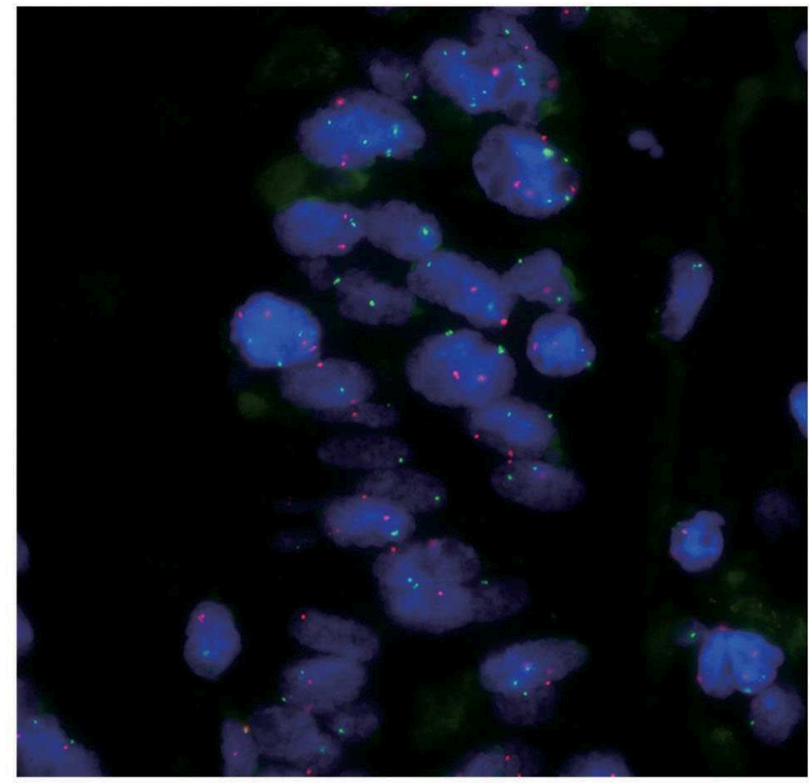

CSP7/CSP3

\section{Case $\mathrm{F}$}

FIGURE 3 | Cytological and histological FISH test results in case F. (A) Microscopy revealed moderately differentiated adenocarcinoma (hematoxylin-eosin staining, magnification×200); (B,C) case F urinary and histological FISH testing was negative; red represents CSP7 and GLPp16, and green represents CSP7 and CSP17.

in more patients. Moreover, studies (17-19) have shown that urinary FISH can be used to predict the recurrence of urothelial carcinoma. Therefore, in theory, FISH may also be useful for urachal carcinoma monitoring after surgery, which requires further research.

\section{CONCLUSION}

In summary, UroVysion FISH was found to be positive in a high proportion of urachal carcinoma of late stage, which is a potential diagnostic indicator of urachal 
carcinoma. Its chromosomal aberrations may be also different from those of urothelial carcinoma, which may aid in their differential diagnosis. Fluorescence in situ hybridization positivity should not be simply interpreted as urothelial carcinoma.

\section{Clinical Practice Points}

- UroVysion FISH is a potential diagnostic indicator of latestage urachal carcinoma with hematuria.

- Fluorescence in situ hybridization can be positive in a number of different diseases presenting with hematuria.

- Chromosomal aberrations of urachal carcinoma seem to be different from those of urothelial carcinoma, which may aid in their differential diagnosis.

\section{DATA AVAILABILITY STATEMENT}

The raw data supporting the conclusions of this article will be made available by the authors, without undue reservation.

\section{ETHICS STATEMENT}

The studies involving human participants were reviewed and approved by Medical Ethical Committee of Tongji Hospital of Huazhong University of Science and Technology. Written informed consent for participation was not required for this study in accordance with the national legislation and the institutional requirements.

\section{REFERENCES}

1. Wilcox DT, Godbole PP, Koyle MA. Pediatric Urology: Surgical Complications and Management [M]. Houston, TX: Blackwell Publishing Ltd (2008). p. 92-7. doi: 10.1002/9781444304183

2. Scabini S, Rimini E, Romairone E, Scordamaglia R, Vallarino L, Giasotto V, et al. Urachal tumour: case report of a poorly understood carcinoma. World J Surg Oncol. (2009) 7:82. doi: 10.1186/1477-7819-7-82

3. Gopalan A, Sharp DS, Fine SW, Tickoo SK, Herr HW, Reuter VE, et al. Urachal carcinoma: a clinicopathologic analysis of 24 cases with outcome correlation. Am J Surg Pathol. (2009) 33:659-68. doi: 10.1097/PAS.0b013e31819aa4ae

4. Johnson DE, Hodge GB, Abdul-Karim FW, Ayala AG. Urachal carcinoma. Urology. (1985) 26:218-21. doi: 10.1016/0090-4295(85)90112-8

5. Kojima T, Nishiyama H, Ozono S, Hinotsu S, Keino N, Yamaguchi A, et al. Clinical evaluation of two consecutive UroVysion fluorescence in situ hybridization tests to detect intravesical recurrence of bladder cancer: a prospective blinded comparative study in Japan. Int J Clin Oncol. (2018) 23:1140-7. doi: 10.1007/s10147-018-1311-6

6. Mischinger J, Guttenberg LP, Hennenlotter J, Gakis G, Aufderklamm S, Rausch S, et al. Comparison of different concepts for interpretation of chromosomal aberrations in urothelial cells detected by fluorescence in situ hybridization. J Cancer Res Clin Oncol. (2017) 143:677-85. doi: 10.1007/s00432-016-2310-5

7. Lavery HJ, Zaharieva B, McFaddin A, Heerema N, Pohar KS. A prospective comparison of UroVysion FISH and urine cytology in bladder cancer detection. BMC Cancer. (2017) 17:247. doi: 10.1186/s12885-0173227-3

8. Dimashkieh H, Wolff DJ, Smith TM, Houser PM, Nietert PJ, Yang J. Evaluation of urovysion and cytology for bladder cancer detection: a study of 1835 paired urine samples with clinical and histologic

\section{AUTHOR CONTRIBUTIONS}

$\mathrm{ZH}$ : project administration and resources. CK: writing-original draft. ZL: data curation and formal analysis. XZ: software and supervision. SL: writing-review and editing. HX: review and editing. CY: conceptualization, funding acquisition, and writing-review and editing. All authors contributed to the article and approved the submitted version.

\section{FUNDING}

This work was supported by the National Natural Science Foundation of China (No. 81702989).

\section{ACKNOWLEDGMENTS}

The authors would like to thank Professor Zhiqiang Chen (Department of Urology, Tongji Hospital, Tongji Medical College, Huazhong University of Science and Technology) for technical assistance in the analysis of the FISH studies.

\section{SUPPLEMENTARY MATERIAL}

The Supplementary Material for this article can be found online at: https://www.frontiersin.org/articles/10.3389/fmed. 2020.00437/full\#supplementary-material

correlation. Cancer Cytopathol. (2013) 121:591-7. doi: 10.1002/cncy. 21327

9. Shan Z, Wu P, Zheng S, Tan W, Zhou H, Zuo Y, et al. Evaluation of upper urinary tract tumors by FISH in Chinese patients. Cancer Genet Cytogenet. (2010) 203:238-46. doi: 10.1016/j.cancergencyto.2010. 07.133

10. Zhou L, Yang K, Li X, Ding Y, Mu D, Li H, et al. Application of fluorescence in situ hybridization in the detection of bladder transitional-cell carcinoma: a multi-center clinical study based on Chinese population. Asian J Urol. (2019) 6:114-21. doi: 10.1016/j.ajur.2018.06.001

11. Kipp BR, Tyner HL, Campion MB, Voss JS, Karnes RJ, Sebo TJ, et al. Chromosomal alterations detected by fluorescence in situ hybridization in urothelial carcinoma and rarer histologic variants of bladder cancer. Am J Clin Pathol. (2008) 130:552-9. doi: 10.1309/DFJUHY3WPC 9GUU2W

12. Yang C, Liu Z, Lan R, Wang Z, Hu Z, Chen Z, et al. Paraganglioma of the urinary bladder with chromosome duplications detected by fluorescence in situ hybridization in urine exfoliated cells: a case report. Oncol Lett. (2016) 11:795-7. doi: 10.3892/ol.2015.3941

13. Reid-Nicholson MD, Ramalingam P, Adeagbo B, Cheng N, Peiper SC, Terris MK. The use of Urovysion fluorescence in situ hybridization in the diagnosis and surveillance of non-urothelial carcinoma of the bladder. Mod Pathol. (2009) 22:119-27. doi: 10.1038/modpathol.2008.179

14. Szarvas T, Modos O, Niedworok C, Reis H, Szendroi A, Szasz MA, et al. Clinical, prognostic, and therapeutic aspects of urachal carcinoma-A comprehensive review with meta-analysis of 1,010 cases. Urol Oncol. (2016) 34:388-98. doi: 10.1016/j.urolonc.2016.04.012

15. Lopez-Beltran A, Requena MJ, Cheng L, Montironi R. Pathological variants of invasive bladder cancer according to their suggested clinical significance. BJU Int. (2008) 101:275-81. doi: 10.1111/j.1464-410X.2007. 07271.x 
16. Ashley RA, Inman BA, Sebo TJ, Leibovich BC, Blute ML, Kwon ED, et al. Urachal carcinoma: clinicopathologic features and long-term outcomes of an aggressive malignancy. Cancer. (2006) 107:712-20. doi: 10.1002/cncr. 22060

17. Gofrit ON, Zorn KC, Silvestre J, Shalhav AL, Zagaja GP, Msezane LP, et al. The predictive value of multi-targeted fluorescent in-situ hybridization in patients with history of bladder cancer. Urol Oncol. (2008) 26:246-9. doi: 10.1016/j.urolonc.2007.02.011

18. Daniely M, Rona R, Kaplan T, Olsfanger S, Elboim L, Freiberger A, et al. Combined morphologic and fluorescence in situ hybridization analysis of voided urine samples for the detection and follow-up of bladder cancer in patients with benign urine cytology. Cancer. (2007) 111:517-24. doi: $10.1002 /$ cncr.23119

19. Skacel M, Fahmy M, Brainard JA, Pettay JD, Biscotti CV, Liou LS, et al. Multitarget fluorescence in situ hybridization assay detects transitional cell carcinoma in the majority of patients with bladder cancer and atypical or negative urine cytology. J Urol. (2003) 169:2101-5. doi: 10.1097/01.ju.0000066842.45464.cc

Conflict of Interest: The authors declare that the research was conducted in the absence of any commercial or financial relationships that could be construed as a potential conflict of interest.

Copyright $\odot 2020 \mathrm{Hu}, \mathrm{Ke}, \mathrm{Liu}, \mathrm{Zeng}, \mathrm{Li}, \mathrm{Xu}$ and Yang. This is an open-access article distributed under the terms of the Creative Commons Attribution License (CC BY). The use, distribution or reproduction in other forums is permitted, provided the original author(s) and the copyright owner(s) are credited and that the original publication in this journal is cited, in accordance with accepted academic practice. No use, distribution or reproduction is permitted which does not comply with these terms. 